\title{
A Novel Tool to Facilitate Crimping Suture Placement for a Modified David V/Miller Aortic Root Replacement
}

\author{
Sergey Boldyrev, MD, PhD*, Kirill Barbukhatty, MD, PhD, Vladimir Porhanov, MD, PhD \\ Department of Adult Cardiac Surgery, Regional Clinical Hospital \#1, Krasnodar, Russian Federation
}

\begin{abstract}
Surgical treatment of aortic root and ascending aorta aneurysms with aortic insufficiency is still controversial. A valvesparing operation is the procedure of choice for such patients, and the reimplantation technique is preferable. We describe a simple technique for aortic root reconstruction that has been successfully performed for patients with aneurysms of aortic root and ascending aorta with aortic insufficiency.

Copyright @ 2014 Science International Corp.
\end{abstract}

\section{Key Words}

Aneurysm - Aortic valve - Aortic root - Dissection

\section{Introduction}

Surgical treatment of aortic root and ascending aorta aneurysms with aortic insufficiency is still controversial. A valve-sparing operation is the procedure of choice for such patients, and the reimplantation technique is preferable [1]. Nowadays, multiple modifications of the reimplantation technique have been introduced. We describe a simple technique for aortic root reconstruction that has been successfully performed for patients with aneurysms of aortic root and ascending aorta with aortic insufficiency.

After median sternotomy, cardiopulmonary bypass is begun, using the classic scheme "aorta-right atrium" with moderate hypothermia (to $32^{\circ} \mathrm{C}$ ). After completion of cardioplegia, the aneurysm is resected, leaving approximately $4-5 \mathrm{~mm}$ of the attached aortic wall, and the coronary arteries are excised with buttons. Then, the fibrous annulus is measured and a vascular graft is prepared for reimplantation. For those manipulations we created a new, simple, and convenient device (Fig. 1). There is a circular stop on the opposite side of the device. Cylinders are changeable and are of different diameters: $21,23,25,27$, or $29 \mathrm{~mm}$. The working part of each cylinder has streamlined circular grooves $0.2 \mathrm{~mm}$ deep at a distance of $2 \mathrm{~mm}$ from each other. The fibrous annulus is measured by inserting the device into the left ventricle through the annulus of the aortic valve (Fig. 2).

We then prepare the vascular graft for reimplantation. An oversized vascular graft (annulus diameter $=$ device chosen size $+8 \mathrm{~mm}$ ) is necked down proximally to fit an appropriate device using 2-0 horizontal interrupted polyester plication sutures. The assistant introduces the device into the selected prosthesis to the circular stop. The surgeon performs the first proximal suture line. The first line of the suture is located 1-2 $\mathrm{mm}$ from the proximal edge of the prosthesis. The suture is tightened so that the graft is partially fixed on one of the device's circular grooves. The second line is located 3-4 $\mathrm{mm}$ above the first line. The ribs of sutures are located in a chess order, thereby resulting in a border of approximately $5 \mathrm{~mm}$ (Figs. 3 and 4). The proximal suture line is performed by using 2-0 polyester sutures with pledgets that are passed from inside the left ventricular outflow tract to the outside,

*Corresponding author:

Sergey Boldyrev, MD, PhD

Department of Adult Cardiac Surgery

Regional Clinical Hospital \#1

Krasnodar, Russian Federation

Tel: +7 9184437589, Fax: +7 86125262 90, E-Mail: bolsy@rambler.ru 


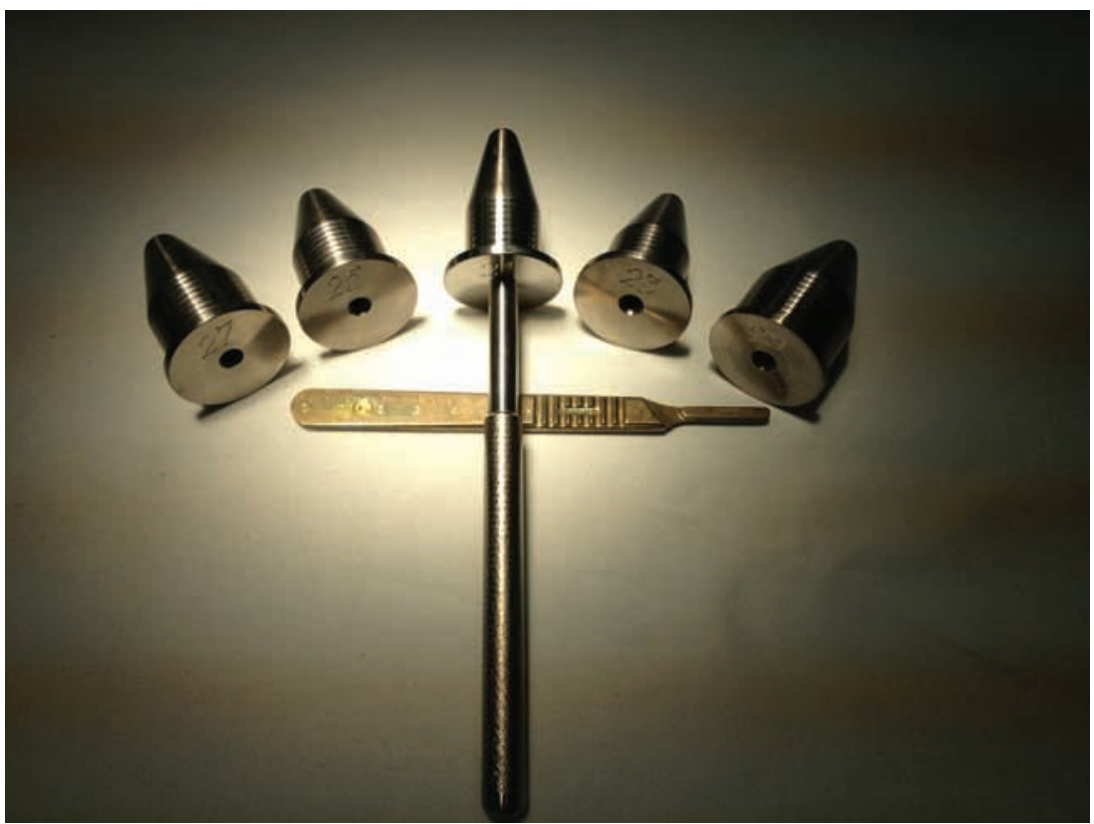

Figure 1. The new device, which has changeable cylinders with different diameters.

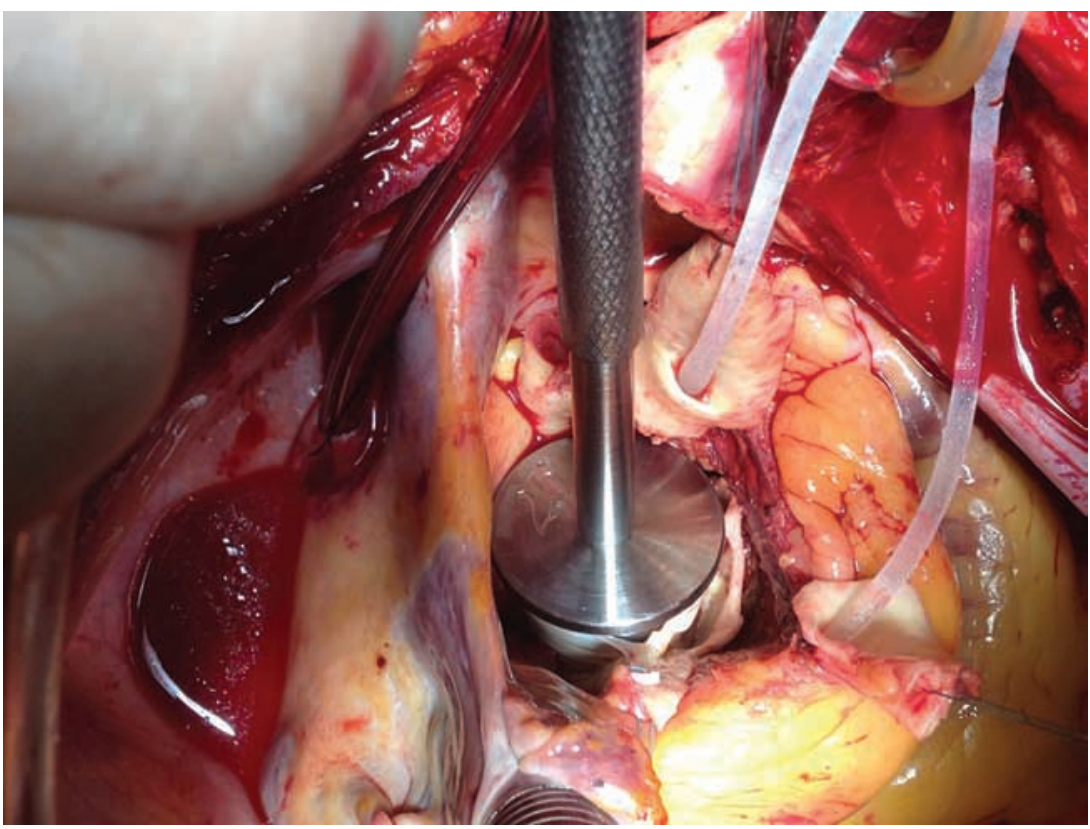

Figure 2. Measuring of fibrous annulus diameter by inserting the new device into the left ventricle through the annulus of the aortic valve.

under the aorta cusps. Then the pledgeted sutures pass through the prosthesis base edge as follows: $1-2$ $\mathrm{mm}$ lower, between, and 1-2 mm higher than the lines formed by two horizontal sutures. Then, the graft is anchored in the aortic root by tying the suture. Thus, a zigzag line of fixation is created along the whole circular length (Fig. 5). Once the graft is anchored in the aortic root, the commissures are trimmed by placing the stay sutures at the appropriate height inside the vascular graft. The correct position of the commissures inside the graft is identified by pulling on both the commissure and the vascular graft before stitching the sutures through the graft. The graft should extend by half or two thirds of its maximum length at this 


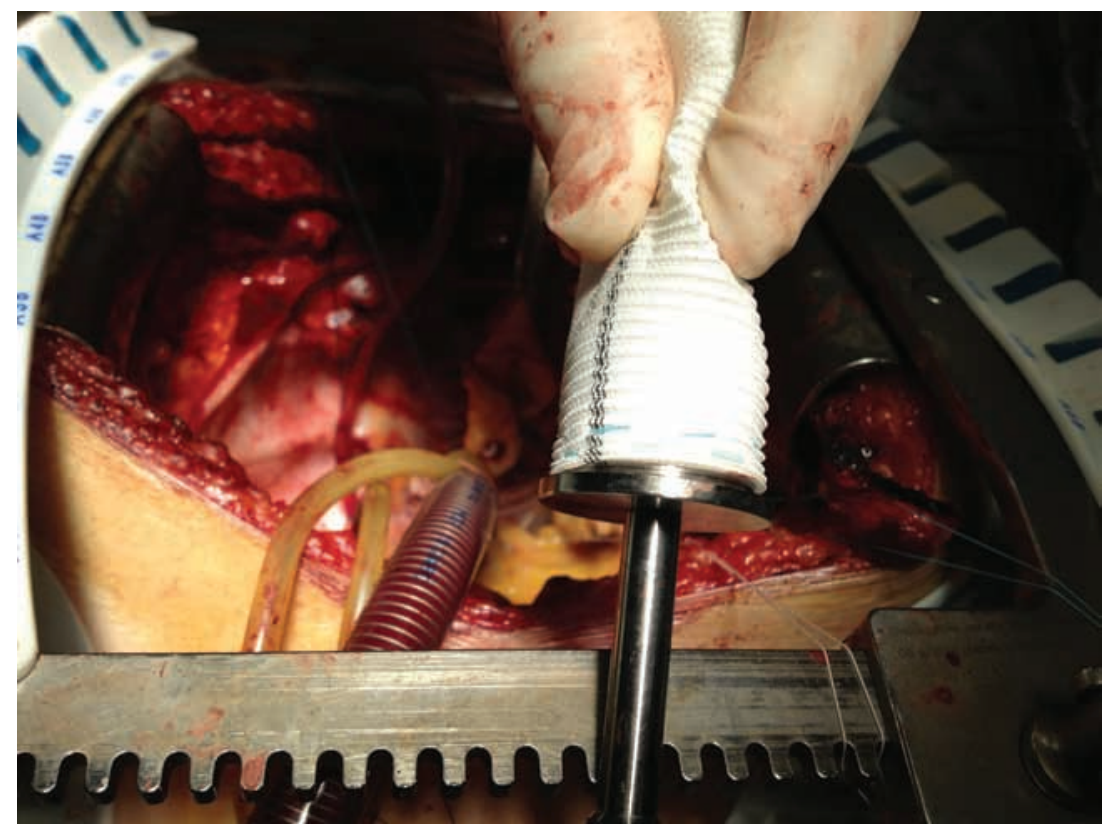

Figure 3. An oversized vascular graft is necked down proximally to fit an appropriate size of the new device using 2-0 interrupted polyester plication sutures. The first line of the suture is located 1-2 $\mathrm{mm}$ from the proximal edge of the prosthesis; the second line is located $3-4 \mathrm{~mm}$ above the first line.

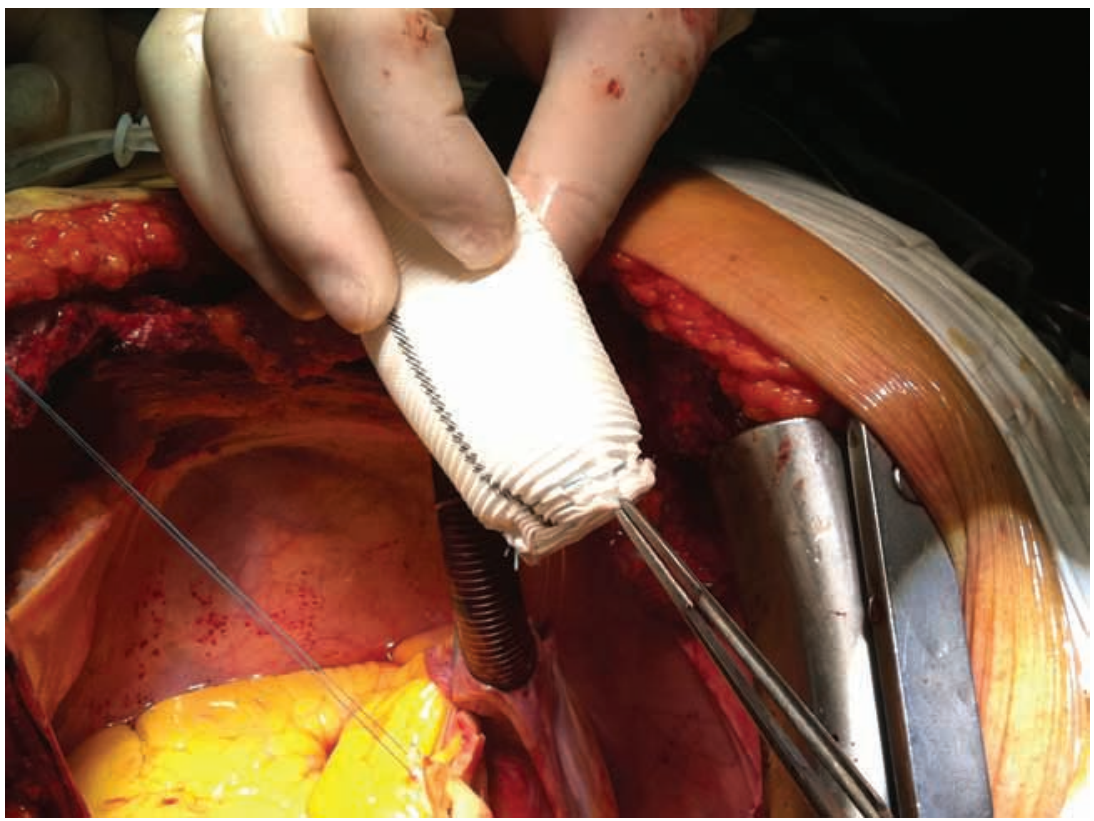

Figure 4. A border of the proximal part of the graft.

segment. When the commissures are trimmed, the tissue remnants of the partially resected sinuses of Valsalva are reimplanted into the vascular graft using 4-0 monofilament running sutures. The aortic root is finally formed by 2-0 polyester-interrupted or plication sutures at the sinotubular ridge level (Fig. 6).

\section{Clinical Experience}

Between 2011 and 2013, a total of 39 patients (mean age $49 \pm 17$ years, range 14-70 years; 30 men) with aneurysms of the aortic root and ascending aorta and aortic insufficiency underwent aortic root reconstruction. All patients had aortic regurgitation; 24 pa- 


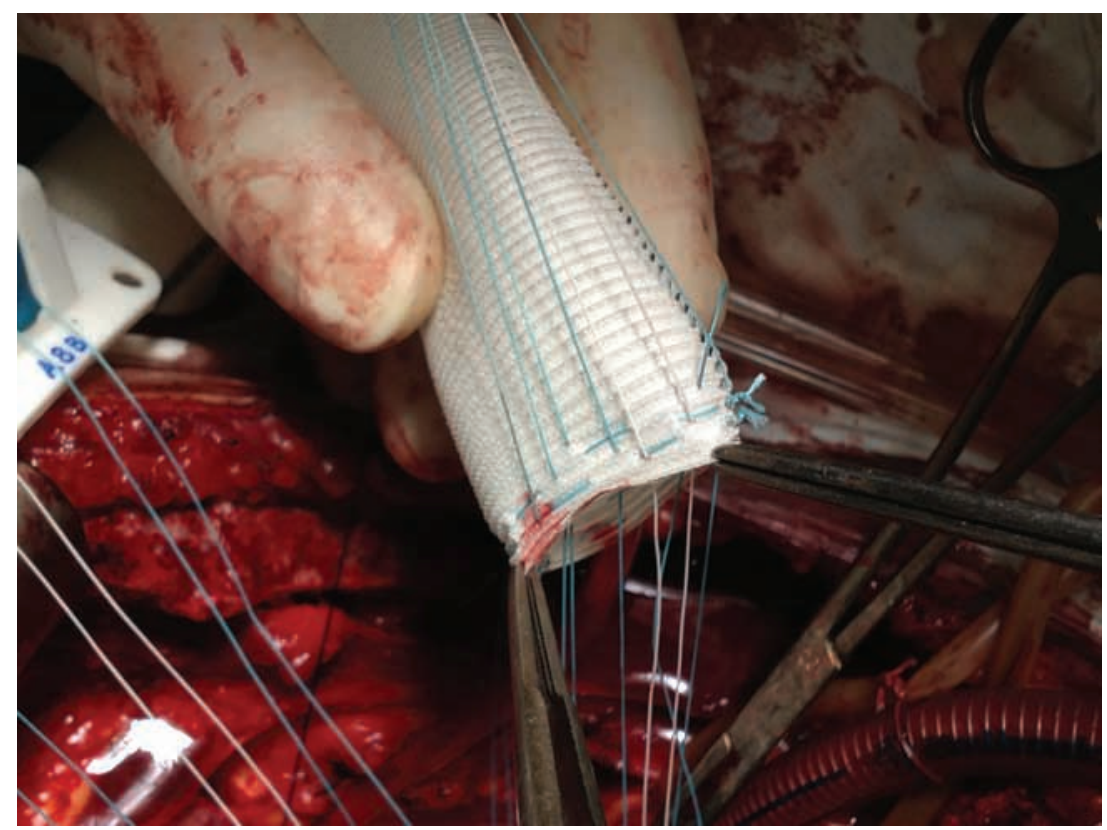

Figure 5. The zigzag line of fixation.

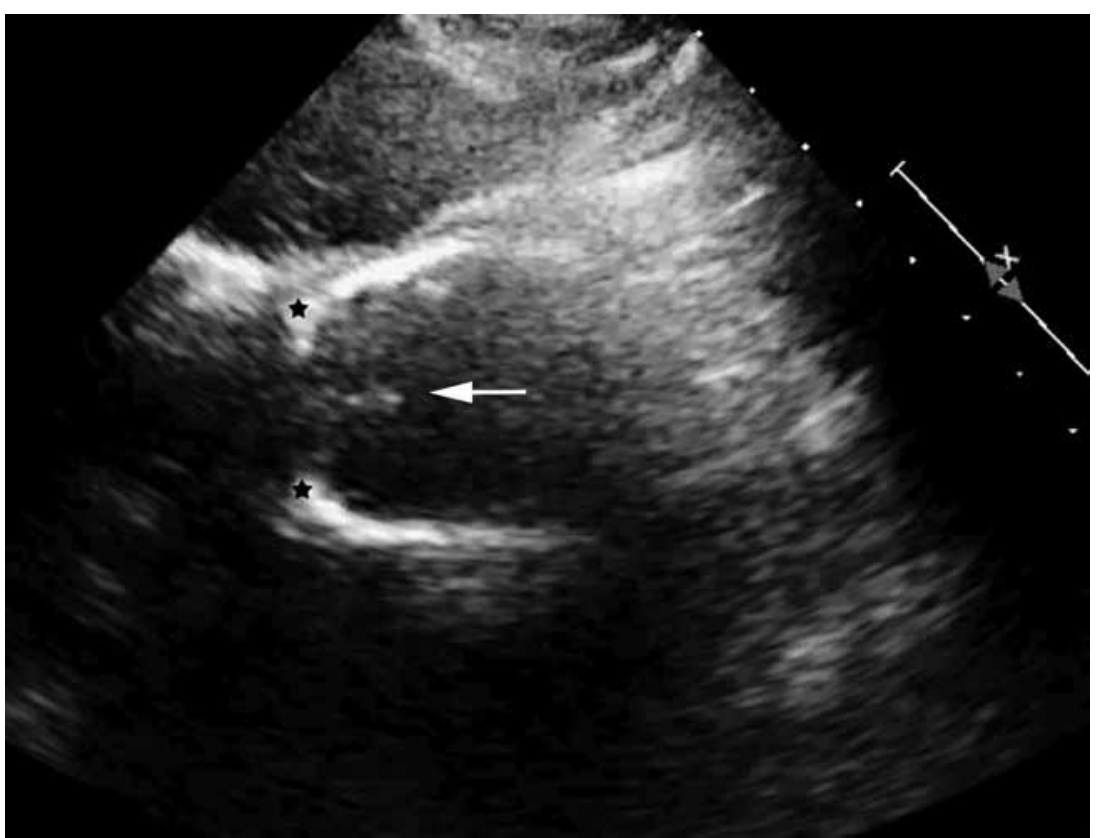

Figure 6. Echocardiography scan of the aortic root, the coaptation zone (arrow), and the bracing ring (stars).

tients had $3+$ insufficiency. Before the procedure, the end-diastolic diameter of the left ventricle (EDDLV) was $55.3 \pm 6.9 \mathrm{~mm}(44-69 \mathrm{~mm})$, and the ejection fraction (EF) was $53.5 \pm 6.8 \%(34-65 \mathrm{~mm})$. There were no complications related to the aortic valve and root reconstruction observed. All patients survived the surgery and were discharged from the hospital (average, 12 days after surgery). The mean gradient across the valve was $7.8 \pm 2.6 \mathrm{~mm} \mathrm{Hg}$. After the procedure, EDDLV was $52.8 \pm 6.7 \mathrm{~mm}(43-65 \mathrm{~mm})$, and $\mathrm{EF}$ was $49.1 \pm 8 \%$ (30-59 $\mathrm{mm})$. The follow-up extended to 24 months after the operation, and echocardiography results showed no significant aortic valve regurgitation in 34 patients, and a moderate level (grade 2) in 5 patients. 


\section{Additional Surgical Procedures}

A total of 11 patients underwent "hemiarch" replacement of the aortic arch, 8 had aortic valve leaflet reconstruction, 4 had concomitant coronary artery bypass grafting, and 1 had reconstruction of the mitral valve.

\section{Discussion}

Creation of novel sinuses of Valsalva is the key point of any reimplantation technique for long-term functioning of the new aortic root. Despite the fact that some clinics apply the reimplantation technique (David I) with satisfactory results [2], the overwhelming majority of surgeons prefer to construct novel sinuses in a novel aortic root. The importance of the mechanism of opening and closing of the aortic valve was depicted by Leonardo da Vinci in the 15th century. Risk of damage to the leaflets in a narrow prosthesis without sinuses makes surgeons strive to recreate the original anatomy. The reimplantation technique requires very careful selection of graft size. This topic is the subject of many publications, paying tribute to the operation's complexity. The Seattle modification partially solved the problem of creation of new sinuses [3], but the Miller modification [4] simplified reimplantation of the aortic valve. At the beginning of our experience we used a one-line plication suture for narrowing the graft's proximal part, as described by Dr. Miller. Purposely, we used a graft $8 \mathrm{~mm}$ bigger than the native aortic annulus. With this approach, manipulations inside the prosthesis become free. However, the graft receives a significant proximal corrugation. We concluded that one line of plication sutures is unreliable for the anastomosis to hold pressure subsequently. Its strength properties are not sufficient to maintain the shape of the aortic root and to prevent bleeding despite the fact that there are fixing sutures with pledgets going from the left ventricular outflow tract outward. Thus, using two suture lines allows creation of a border of approximately 5 $\mathrm{mm}$. The Miller technique supposes fixation of the graft at the horizontal line. We offer a zigzag line of fixation.

There are several reasons to use the zigzag technique and our new device. First, this technique allows one to minimize risk of bleeding. In our experience we have had one complication that led to gushing bleed- ing from the anastomosis zone after reestablishment of cardiac function. We managed to fix this problem despite some technical complexity. After this, we started to use our zigzag technique and did not encounter subsequent similar complications. Second, our narrowing of the proximal part of the graft creates crimping like the Miller technique does. The zigzag technique helps us to construct a thick line of contacting surface between the prosthesis and the left ventricular outflow tract. In addition, the zigzag technique helps to reduce excessive plication of fibrous annulus. From the point of view of physics, the advantage of our approach can be justified as follows. Every horizontal filament at the proximal graft has ribs of 5-7 $\mathrm{mm}$ lying in turn on the front and back sides. Every rib after its final fixation cements the surface area coaptation. Third, the bracing ring prevents dilatation of the aortic annulus. Fourth, in the beginning of our experience we used valve sizers, but we encountered some problems with sliding of the graft along the plastic sizer during narrowing. We introduced the new device (Patent Pending \# 2013107839) and achieved the following advantages: a circular stop helps to fix the graft, and shallow circular grooves prevent the graft from sliding when fixing filaments.

\section{Conclusion}

Our approach helps to reconstruct a natural analog of the aortic root, to stabilize the aortic annulus, and to minimize risk of bleeding. This technique is quick, simple, and reproducible with multiple grafts.

\section{Acknowledgments}

This work was presented at the 63th International Congress of The European Society for Cardiovascular and Endovascular Surgery (ESCVS), Nice, France, 24-27 April 2014.

\section{Conflict of Interest}

The authors have no conflict of interest relevant to this publication.

Comment on this Article or Ask a Question 


\section{References}

1. Hiratzka LF, Bakris GL, Beckmann JA, Bersin RM, Carr VF, Casey DE Jr, et al. 2010 ACCF/AHA/ AATS/ACR/ASA/SCA/SCAI/SIR/STS/SVM guidelines for the diagnosis and management of patients with thoracic aortic disease. Circulation. 2010;121: e266-e369. 10.1161/CIR.0b013e3181d4739e

2. Shrestha M, Baraki H, Maeding I, Fitzner S, Sarikouch S, Khaladj N, et al. Long-term results after aortic valve-sparing operation (David I). Eur J Cardiothorac Surg. 2012;41:56-61. 10.1016/j.ejcts.2011.04.012
3. Cochran RP, Kunzelman KS, Eddy A, Hofer BO, Verrier ED. Modified conduit preparation creates a pseudosinus in an aortic valve-sparing procedure for aneurysm of the ascending aorta. J Thorac Cardiovasc Surg. 1995;109:1049-1057. 10.1016/S00225223(95)70187-7

4. Miller D. Valve-sparing aortic root replacement in patients with the Marfan syndrome. J Thorac Cardiovasc Surg. 2003;125:773-778. 10. 1067/mtc.2003.162
Cite this article as: Boldyrev $S$ Barbukhatty K, Porhanov V. A Novel Tool to Facilitate Crimping Suture Placement for a Modified David V/Miller Aortic Root Replacement. Aorta 2014;2(4): 161-166. DOI: http://dx.doi.org/10.12945/ j.aorta.2014.14-024 